\title{
Is It Time for Gout Flare Treatment to Move into the 21st Century?
}

Advances in science have offered opportunities to develop targeted therapies for multiple disease states. Physicians and patients are becoming accustomed, and even expect, to use targeted therapies, avoiding any "shotgun approaches." The discovery of biologics has revolutionized the treatment of rheumatological diseases. The benefits have greatly outweighed the risks with biologic therapy, and have subsequently reduced tender and swollen joints, improved quality of life, decreased morbidity, and slowed disease progression in this population as a whole ${ }^{1}$.

But despite our greater understanding of the physiology of the inflammatory process of gout, our approach to treatment has remained antiquated. The approved US Food and Drug Administration (FDA) therapies for gout flares include indomethacin, naproxen, sulindac, corticosteroids, and colchicine $e^{2,3,4,5}$. Nonsteroidal antiinflammatory drugs (NSAID) have been used to treat gout flares since the 1960s, corticosteroids since the $1950 \mathrm{~s}$, and documented use of colchicine goes back to $1763^{2,6}$. The efficacy of all these medications for the treatment of gout flares is well documented, but not all patients respond adequately; and in a population that is likely to have 1 or more comorbid conditions, these FDA-approved medications can carry significant risks and adverse effects ${ }^{7}$.

In this issue of The Journal, Desmarais and Chu evaluate the efficacy and safety of anakinra, a biologic therapy that targets interleukin (IL)-1 receptors, thereby blocking IL-1 activity, a major driver of inflammation in an acute gout flare $^{8}$. The authors used retrospective data spanning almost 9 years from the Oregon Health \& Science University (OHSU) Hospital and the Veteran's Administration Portland Health Care System (VAPORHCS) to identify hospitalized patients who carried a diagnosis of gout or calcium pyrophosphate (CPP) deposition, who flared, and who had received at least one 100-mg dose of anakinra while an inpatient. Data analyzed were patient characteristics, comorbidities, reason for anakinra use, number of doses of anakinra, adverse events, functional improvement, and also as importantly, the patients' pain response using a $0-10$ visual analog scale (VAS).

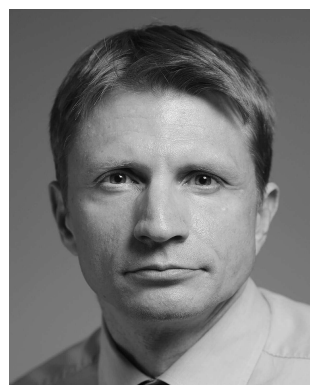

The study provides a real-world snapshot of inpatient gout flares and the challenges clinicians have in managing a sicker, hospitalized population of patients, compared to an ambulatory or clinical trial population. Between the 2 sites, Desmarais and Chu identified a total of 91 patients and 115 flares of crystalline arthritis, with 77 of those patients with 98 gout flares treated with anakinra. Eleven patients with 14 CPP flares, and 3 patients considered to have both gout and CPP flares were also treated with anakinra. More than half of the patients had at least 1 comorbid condition such as diabetes, chronic kidney injury, or congestive heart failure that influenced the treatment decision making of their provider $^{9}$. As expected, the majority were male and their average age was 65 years between the 2 sites. Impressively, $95 \%$ and $88 \%$ of the OHSU and VAPORHCS patients, respectively, were urate-crystal proven. Many of the flares were oligo-to-poly-articular and the average number of joints involved was 4 across both hospitals, which could imply a patient population with a greater severity of disease and crystal burden than the "average" patient with crystalline arthritis ${ }^{10}$.

Regarding flare treatment, the authors noted that some patients received anakinra as a first-line treatment, but most had received other therapies prior to the initiation of anakinra. In patients with gout flare, $40 \%$ received prednisone, $39 \%$ received colchicine, $15 \%$ received intraarticular corticosteroid injections, and 5\% received NSAID. In CPP arthritis flare patients, $14 \%$ received prednisone, $14 \%$ colchicine, $14 \%$ intraarticular injection, and 36\% NSAID. The clinical response to therapy was determined by a pain scale response improvement of 50\% on the VAS in addition to functional improvement. Patients who did not have a VAS improvement or clinical response were considered nonresponders.

For the 77 patients with 98 flares treated with anakinra, the average time period of treatment for the 2 sites was 4 days. Ninety-two percent of the flares had an unequivocal response by Day 2. Of those with clear responses, $37 \%$ had a VAS improvement of $50 \%$ one day after the anakinra was started. There were only 11 patients with 14 CPP arthritis,

See Anakinra in crystalline diseases, page 748

Personal non-commercial use only. The Journal of Rheumatology Copyright (C) 2019. All rights reserved. 
but in this small group, $79 \%$ responded to anakinra, requiring 2-4 doses for each flare episode. Impressively, of the 88 hospitalized patients receiving anakinra, only 1 reported an adverse event, described as a rash that occurred on Day 3 that was treated with antihistamines and did not interrupt therapy.

To date, this is the largest retrospective study evaluating the efficacy and safety of anakinra for acute crystalline arthritis in hospitalized patients. Similar outcomes were also observed in smaller observational studies both in the United States and abroad. For example, Thueringer, et al reported 13 critically ill patients with acute flares who received anakinra because of contraindications to traditional therapies ${ }^{11}$. Half of the patients had a total resolution or marked improvement of their flare at $24 \mathrm{~h}, 40 \%$ responded by $48 \mathrm{~h}$, and the remaining $10 \%$ by $72 \mathrm{~h}$. Ghosh, et al reported on the safety and efficacy of 26 complex hospitalized patients with 40 gout flares treated with anakinra ${ }^{12}$. Comorbid conditions in the retrospective cohort ranged from acute leukemia patients to solid organ transplant patients. Sixty-seven percent of the 40 flares had a significant response by $24 \mathrm{~h}$, while $18 \%, 5 \%$, and $10 \%$ responded in 25-48 h, 49-72 h, and > $72 \mathrm{~h}$, respectively. Loustau, et al reported on the use of anakinra in 31 gout patients with stage $4-5$ chronic kidney injury or kidney transplant ${ }^{13}$. This study of high-risk patients also showed meaningful responses, with only 1 infection in a patient who was taking 3 months of continuous anakinra therapy.

There is a paucity of literature regarding CPP arthritis, but recently, the use of anakinra was reported from a single-center retrospective study including 33 patients ${ }^{14}$. The majority of the patients had oligo-to-poly-articular flares. Six patients had contraindications to standard therapy, and so received anakinra as first-line therapy. The authors found that VAS pain scores decreased by over $50 \%$ by Day 4 , while tender joint counts and CRP decreased by around $75 \%$.

In addition to retrospective studies, there are published randomized clinical trial data using IL-1 inhibition in gout flares. Canakinumab and rilonacept, both IL- $1 \beta$ traps ${ }^{15}$, have been studied in outpatients for the prevention or treatment of flares as either a primary or secondary outcome in randomized, multicenter, active-controlled, double-blind trials ${ }^{16,17}$. Rilonacept did not prove to be more efficacious than indomethacin, but canakinumab was approved by the European Medicines Agency for the treatment of acute gout. Unfortunately, the FDA's Arthritis Advisory Committee denied approval of its use, even though it recognized an unmet need for the treatment of flares ${ }^{18}$. The committee's concern was the safety of IL-1 inhibition in a sicker population whose condition is not life-threatening ${ }^{18}$.

Despite the inherent limitations of Desmarais and Chu's retrospective study, their contribution to the literature is important. They not only showed how efficacious anakinra is for acute flares of crystalline arthritis, but also its safety in a large number of hospitalized patients with multiple comorbid conditions ${ }^{8}$. The patient baseline characteristics in the study were "typical" of the patient with gout we may see in consultation, but would not necessarily qualify for a randomized clinical trial. Around half of the patients had diabetes, chronic kidney injury, or heart failure, or a combination thereof. The real-world applicability of the study is high, given that the patient sample is from both a university and a Veterans Administration medical center, so some inference could be made to similar institutions around the country. Also, the data recorded was another strength of the study. Despite the obstacle to determining the VAS pain retrospectively in this study, the VAS for pain is considered the most important core measurement for clinical gout flare studies by OMERACT ${ }^{19}$, and those patients with incomplete data were considered nonresponders to minimize the risk of overreporting any positive results. The importance of pain relief should not be underestimated in gout studies. Whether it is a randomized, controlled, double-blinded, clinical trial, or a retrospective chart review, pain relief should be a core measurement. Pain, along with tender and swollen joints, is what affects the patient's quality of life and the ability to go to work or to do physical therapy in the hospital, and may be the patient's focus rather than the reason for their admission to the hospital. Persistent gout flares have shown to increase the length of hospital stays ${ }^{20}$, thereby delaying physical therapy or even treatment for the underlying admission diagnosis. This delay is not only bad for the patient, but costly for our health system.

Desmarais and Chu noted that non-rheumatologists, likely internists and hospitalists, were also comfortable prescribing anakinra for gout flares under certain conditions, such as that the patient had previously tolerated it and it had shown efficacy. Also, some patients received anakinra as first-line therapy, when the risks of anakinra were less than those of traditional therapies. This implies a certain amount of comfort with using anakinra by both rheumatologists and non-specialists alike. It also addresses their thought process when deciding how to manage gout flares given a set of risks and benefits in this higher-risk population. It also potentially provides insight regarding the importance of decreasing pain, improving mobility, improving patient quality of life, and even discharge planning in physician decision making.

In this issue of The Journal, Desmarais and $\mathrm{Chu}^{8}$ made a substantial contribution to the literature regarding the safety and efficacy of the use of anakinra for gout flares in patients with multiple comorbid conditions who are representative of patients we see in everyday practice. The authors provide evidence that patients who are older, at higher risk for infection, and have multiple comorbid conditions can receive IL-1 inhibitors safely and effectively for their gout flares. Is it time that gout flare treatment moved into the 21 st century, and stopped being so antiquated?

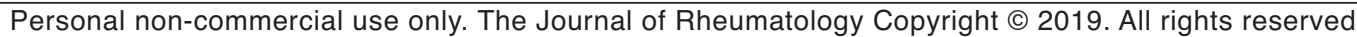


ROBERT T. KEENAN (D), MD, MPH, MBA, Division of Rheumatology,

Department of Medicine,

Duke University School of Medicine,

Durham, North Carolina, USA.

Address correspondence to Dr. R.T. Keenan, Duke University School of Medicine, 200 Trent Drive, DUMC 3544, Durham, North Carolina 27710, USA.E-mail: Robert.keenan@duke.edu

\section{REFERENCES}

1. Kavanaugh A, Singh R, Karki C, Etzel CJ, Kremer JM, Greenberg $\mathrm{JD}$, et al. Disease activity and biologic use in patients with psoriatic arthritis or rheumatoid arthritis. Clin Rheumatol 2018;37:2275-80.

2. Smyth CJ, Percy JS. Comparison of indomethacin and phenylbutazone in acute gout. Ann Rheum Dis 1973;32:351-3.

3. Willkens RF, Case JB, Huix FJ. The treatment of acute gout with naproxen. J Clin Pharmacol 1975;15:363-6.

4. Karachalios GN, Donas G. Sulindac in the treatment of acute gout arthritis. Int J Tissue React 1982;4:297-9.

5. Slobodnick A, Shah B, Pillinger MH, Krasnokutsky S. Colchicine: old and new. Am J Med 2015;128:461-70.

6. Nuki G, Simkin PA. A concise history of gout and hyperuricemia and their treatment. Arthritis Res Ther 2006;8 Suppl 1:S1.

7. Keenan RT, O'Brien WR, Lee KH, Crittenden DB, Fisher MC, Goldfarb DS, et al. Prevalence of contraindications and prescription of pharmacologic therapies for gout. Am J Med 2011;124:155-63.

8. Desmarais J, Chu CQ. Utility of anakinra in acute crystalline diseases: a retrospective study comparing a university hospital with a Veterans Affairs medical center. J Rheumatol 2019;46:748-50.

9. Elfishawi MM, Zleik N, Kvrgic Z, Michet CJ Jr., Crowson CS, Matteson EL, et al. The rising incidence of gout and the increasing burden of comorbidities: a population-based study over 20 years. J Rheumatol 2018;45:574-9.

10. Khanna PP, Nuki G, Bardin T, Tausche AK, Forsythe A, Goren A, et al. Tophi and frequent gout flares are associated with impairments to quality of life, productivity, and increased healthcare resource use: results from a cross-sectional survey. Health Qual Life Outcomes 2012;10:117.
11. Thueringer JT, Doll NK, Gertner E. Anakinra for the treatment of acute severe gout in critically ill patients. Semin Arthritis Rheum 2015;45:81-5.

12. Ghosh P, Cho M, Rawat G, Simkin PA, Gardner GC. Treatment of acute gouty arthritis in complex hospitalized patients with anakinra. Arthritis Care Res 2013;65:1381-4.

13. Loustau C, Rosine N, Forien M, Ottaviani S, Juge PA, Lioté F, et al Effectiveness and safety of anakinra in gout patients with stage 4-5 chronic kidney disease or kidney transplantation: A multicentre, retrospective study. Joint Bone Spine 2018 Apr 11 (E-pub ahead of print).

14. Thomas M, Forien M, Palazzo E, Dieudé P, Ottaviani S. Efficacy and tolerance of anakinra in acute calcium pyrophosphate crystal arthritis: a retrospective study of 33 cases. Clin Rheumatol 2018 Aug 25 (E-pub ahead of print).

15. Dubois EA, Rissmann R, Cohen AF. Rilonacept and canakinumab. Br J Clin Pharmacol 2011;71:639-41.

16. Schlesinger N, Mysler E, Lin HY, De Meulemeester M, Rovensky J, Arulmani U, et al. Canakinumab reduces the risk of acute gouty arthritis flares during initiation of allopurinol treatment: results of a double-blind, randomised study. Ann Rheum Dis 2011;70:1264-71.

17. Terkeltaub RA, Schumacher HR, Carter JD, Baraf HS, Evans RR, Wang J, et al. Rilonacept in the treatment of acute gouty arthritis: a randomized, controlled clinical trial using indomethacin as the active comparator. Arthritis Res Ther 2013;15:R25.

18. Lowry F. FDA panel says no to canakinumab for gout attacks. Medscape. [Internet. Accessed November 14, 2018.] Available from: www.medscape.com/viewarticle/745076

19. Singh JA, Taylor WJ, Dalbeth N, Simon LS, Sundy J, Grainger R, et al. OMERACT endorsement of measures of outcome for studies of acute gout. J Rheumatol 2014;41:569-73.

20. Fisher MC, Pieper C, Hassell N, Levens J, Fernandez M, Zacker C, et al. Incidence of inpatient acute gouty arthritis flare and impact on length of hospital stay. Ann Rheum Dis 2012;71:1.

J Rheumatol 2019;46:667-9; doi:10.3899/jrheum.181249 